ApJ LeTter, ACCEPTED

Preprint typeset using $\mathrm{IATE}_{\mathrm{E}} \mathrm{X}$ style emulateapj v. 08/13/06

\title{
THE COMPACT GROUP-FOSSIL GROUP CONNECTION: OBSERVATIONS OF A MASSIVE COMPACT GROUP AT Z $=0.22^{1}$
}

Claudia L. Mendes de Oliveira

Departamento de Astronomia, Instituto de Astronomia, Geofísica e Ciências Atmosféricas, Universidade de São Paulo, Rua do Matão 1226, Cidade Universitaria, 05508-090, São Paulo, Brazil; oliveira@astro.iag.usp.br

AND

Eleazar R. CARrasco

Gemini Observatory, Southern Operations Center, AURA, Casilla 603, La Serena, Chile; rcarrasco@gemini.edu ApJ Letter, accepted

\begin{abstract}
It has been suggested that fossil groups could be the canibalized remains of compact groups, that lost energy through tidal friction. However, in the nearby universe, compact groups which are close to the merging phase and display a wealth of interacting features (such as HCG 31 and HCG 79) have very low velocity dispersions and poor neighborhoods, unlike the massive, cluster-like fossil groups studied to date. In fact, known $\mathrm{z}=0$ compact groups are very seldom embedded in massive enough structures which may have resembled the intergalactic medium of fossil groups. In this paper we study the dynamical properties of CG6, a massive compact group at $\mathrm{z}=0.220$ that has several properties in common with known fossil groups. We report on new $g^{\prime}$ and $i^{\prime}$ imaging and multi-slit spectroscopic performed with GMOS on Gemini South. The system has 20 members, within a radius of $1 \mathrm{~h}_{70}^{-1} \mathrm{Mpc}$, a velocity dispersion of $700 \mathrm{~km} \mathrm{~s}^{-1}$ and has a mass of $1.8 \times 10^{14} \mathrm{~h}_{70}^{-1} \mathrm{M}_{\odot}$, similar to that of the most massive fossil groups known. The merging of the four central galaxies in this group would form a galaxy with magnitude $M_{r^{\prime}} \sim-23.4$, typical for first-ranked galaxies of fossil groups. Although nearby compact groups with similar properties to CG 6 are rare, we speculate that such systems occurred more frequently in the past and they may have been the precursors of fossil groups.

Subject headings: galaxies: clusters: individual: HCG 31, HCG 79, HGC 92, SDSS CG 6 - galaxies: kinematics - galaxies: structure
\end{abstract}

\section{INTRODUCTION}

Groups of galaxies are small systems of typically a few $\mathrm{L}^{*}$ galaxies, which comprise over $55 \%$ of the nearby structures in the universe. A small fraction of galaxy groups are classified as compact groups, which are responsible for $\sim 1 \%$ of the luminosity density of the universe (Mendes de Oliveira \& Hickson 1991). Although they are rare objects in the nearby universe, their high galactic densities and low velocity dispersions make them ideal systems for the study of galaxy transformation through galaxy-galaxy collisions. As expected, these systems have a high fraction of interacting members, although merged objects are rare (Zepf 1993). They are commonly believed to evolve through dynamical friction and finally merge to form one single galaxy (Barnes 1992). Vikhlinin et al. (1999) and Jones et al. (2003) have suggested that the merging of compact groups can lead to the formation of fossil groups. A fossil group (FG, hereafter) is a system with an extended and luminous Xray halo $\left(\mathrm{L}_{X}>10^{42} \mathrm{~h}_{70}^{-2} \mathrm{erg} \mathrm{s}^{-1}\right)$, dominated by one single brighter than $\mathrm{L}^{*}$ elliptical galaxy, surrounded by low-luminosity companions (where the difference in mag-

\footnotetext{
${ }^{1}$ Based on observations obtained at the Gemini Observatory, which is operated by the Association of Universities for Research in Astronomy, Inc., under a cooperative agreement with the NSF on behalf of the Gemini partnership: the National Science Foundation (United States), the Particle Physics and Astronomy Research Council (United Kingdom), the National Research Council (Canada), CONICYT (Chile), the Australian Research Council (Australia), CNPq (Brazil) and CONICET (Argentina) - Program ID: GS-2005B-Q-37
}

nitude between the bright dominant elliptical and the next brightest companion is $>2 \mathrm{mag}$ in the R-band; Jones et al. 2003).

One important goal of this article is to investigate if compact groups (CG, hereafter) as we known them today, could be the precursors of FGs. In order to answer this question, we summarize, in section 2 , the properties of a few of the most strongly interacting nearby CGs known, which are about to merge, and in section 3 , we describe the properties of the five FGs which have been studied spectroscopically so far. In section 4 , we present new observations for a CG embedded in a cluster-size potential, at redshift $\mathrm{z}=0.22$ and section 5 puts together all the observations described and discusses the CG-FG scenario. Throughout this paper we adopt when necessary a standard cosmological model: $H_{0}=70 h_{70} \mathrm{~km} \mathrm{~s}^{-1} \mathrm{Mpc}^{-1}$, $\Omega_{m}=0.3$ and $\Omega_{\Lambda}=0.7$. At $\mathrm{z}=0.22,1^{\prime \prime}$ corresponds to $3.5 h_{70}^{-1} \mathrm{kpc}$.

\section{INTERACTING COMPACT GROUPS: HCG 31, HCG 79 AND HCG 92}

There is evidence from both observations and simulations that groups evolve through dynamical friction and coalesce to form more compact structures as the universe ages. A few of the most compact, and therefore most evolved groups known, from Hickson's catalogue (Hickson et al. 1992) are HCG 31, HCG 79 (or Seyfert Sextet) and HCG 92 (or Stephan's quintet). The study of these groups is important to help understanding processes common in merging systems, environments that may have occurred more often in the high-redshift uni- 
verse.

HCG 31 is a group at $\mathrm{z} \sim 0.013$ and with a velocity dispersion of $\sigma \sim 60 \mathrm{~km} \mathrm{~s}^{-1}$. This is a gasrich group with intense star forming activity (e.g. Mendes de Oliveira et al. 2006b; Amram et al. 2007), dominated by a central pair of interacting dwarf galaxies $\mathrm{A}+\mathrm{C}$. HCG 31 is thought to be in a pre-merging phase (Amram et al. 2004; Verdes-Montenegro et al. 2005) and it has well developed tidal tails seen in $\mathrm{H} \alpha$ and $\mathrm{HI}$. The group hosts two excellent candidates for tidal dwarf galaxies, namely member $\mathrm{F}$, in the southern tail and member $\mathrm{R}, 50 \mathrm{~h}_{70}^{-1} \mathrm{kpc}$ to the north of the group (for an assumed distance modulus of $\mathrm{DM}=33.8$ ).

HCG 79, also known as Seyfert Sextet, was originally identified as a sextet of galaxies but it is now known to be a quartet at $\mathrm{z}=0.0145$ (the 5 th object is in the background and the 6 th is a luminous tidal debris to the northeast of the group). This is the most CG in Hickson's catalogue with a galaxy-galaxy distance below 10 $\mathrm{kpc}$ (for an adopted $\mathrm{DM}=34.0$ ) and a velocity dispersion of $\sigma=138 \mathrm{~km} \mathrm{~s}^{-1}$. The four galaxies present morphological distortions and increased activity (tidal debris, bar in HCG 79B, dust lane in HCG 79A, radio and infrared emission, disturbed rotation curves and nuclear activity). The group presents a prominent intra-group light envelope which contains $45 \%$ of the total light of the group (Da Rocha and Mendes de Oliveira 2005) and irregular envelopes of HI (Williams et al. 1991) and Xrays (Pildis et al. 1995). These suggest that recent or on-going interaction is taking place within this system.

HCG 92, also known as Stephan's quintet, is in reality a quartet with $\mathrm{z}=0.0215$ and a foreground galaxy. It is the most well studied CG - multi wavelength data are available from radio to X-rays. Most of the gaseous material in Stephan's quintet is concentrated not in the galaxies but in the intragroup medium, suggesting that collisions among group members may have happened frequently. A number of tidal dwarf galaxies and intergalactic HII regions have been identified in this group (e.g. Mendes de Oliveira et al. 2001, 2004; Xu et al. 2005). Of the three groups described above, HCG 92 is the only one to have detected $\mathrm{X}$-rays, with a total bolometric luminosity of $2.96 \times 10^{42} \mathrm{~h}_{70}^{-2} \mathrm{erg} \mathrm{s}^{-1}$ (Xue \& Wu 2000).

These three spiral-rich groups are thought to be in their final stages of evolution - they are, in fact, some of the most compact systems found in the Hickson's catalogue. Yet, they have members that can be clearly identified as individual galaxies, suggesting that once merging starts, it may proceed quickly, and the groups may no longer be recognized as such. The bright members of these groups will almost certainly end up as a single galaxy pile. A discussion of whether these systems will most likely end up as FGs or as single isolated elliptical galaxies is deferred to section 5. In the following section, some of the optical properties of the FGs studied so far are summarized.

\section{DYNAMICAL PROPERTIES OF FGS}

Only five FGs have been studied so far in any level of detail in the optical bands (imaging and spectroscopy).

Mendes de Oliveira et al. (2006a) derived the physical properties of the FG RX J1552.2+2013, at a redshift of $\mathrm{z}=0.136$, and computed its luminosity function, based on the spectroscopy of 36 member galaxies. This system was found to be a fossil cluster, given its high number of members and high velocity dispersion (close to 700 $\mathrm{km} / \mathrm{s}$ ).

Cypriano et al. (2006) studied a second FG, RX J1416.4+2315, at a similar redshift of $z=0.137$. For this system also a fairly high velocity dispersion was measured $\left(584 \mathrm{~km} \mathrm{~s}^{-1}\right)$, for 25 members located in the inner $542 \mathrm{~h}_{70}^{-1} \mathrm{kpc}(\sim 0.45$ of the virial radius $)$ of the system. Similar results were found by Khosroshahi et al. (2006).

In two recent studies Mendes de Oliveira et al. (2007, in preparation) and Cypriano et al. (2007, in preparation) two other FGs were also found to have clusterlike masses: RX J1340.5+4017, with 25 members, was found to have a velocity dispersion of $580 \mathrm{~km} \mathrm{~s}^{-1}$ and for RX J1256.0+2556, a velocity dispersion of $582 \mathrm{~km}$ $\mathrm{s}^{-1}$ was determined from spectroscopy of 28 members. In all four cases the systems presented very pronounced red sequences in their color-magnitude diagrams and they were dominated by early-type galaxies. Table 3 of Khosroshahi et al. (2006) lists one other FG for which spectroscopy for a significant number of members has been obtained, ESO 3060170, which is found to have a velocity dispersion of $648 \mathrm{~km} \mathrm{~s}^{-1}$ derived from velocity measurements of 15 members.

The conclusion is then that for all five groups studied so far, for which more than six members are known, they all have velocity dispersions of $\sim 600 \mathrm{~km} \mathrm{~s}^{-1}$ or higher, and dynamical virial masses $\sim 10^{14} \mathrm{~h}_{70}^{-1} \mathrm{M}_{\odot}$.

Fossil groups were suggested to be the end products of merging of $\mathrm{L}^{*}$ galaxies in low-density environments (Jones et al. 2003). However, the five FGs studied do not constitute low-density environments and, in fact, are more similar to galaxy clusters. The fairly high X-ray emission, the large fraction of elliptical galaxies, as well as the lack of obvious substructures, suggest that these FGs are fairly massive virialized systems.

The FGs described above have masses, kinematics and environments which are very different from those of nearby CGs. At first sight, this evidence would seem to indicate that there is no connection between these two kinds of systems. However, before discussing this point any further, we would like to present in the next section, an example of a CG that does have similar optical properties to those of FGs. It is the farthest away CG known to date, at $\mathrm{z}=0.22$. New imaging and spectroscopic observations of this group are presented in the next section. A detail description of the properties of CG 6 will be presented in a future paper (Carrasco et al. 2007, in preparation).

\section{CG 6 - A COMPACT GROUP AT $\mathrm{Z}=0.22$}

CG 6 is a group from the Lee et al. (2004) catalog of CG galaxies, chosen from the SDSS database. Before the present study, only one galaxy in the system, the central one, had a measured redshift. The observations of SDSS CG 6 were carried out using the Gemini Multiobject Spectrograph (hereafter GMOS Hook et al. 2004) at the Gemini South telescope.

The group was imaged using the standards $g^{\prime}$ and $i^{\prime}$ filters (Fukugita et al. 1996) in December $1^{\text {st }}$, 2005. Exposures of $3 \times 150$ seconds in $g^{\prime}$ and $3 \times 120$ seconds in $i^{\prime}$ filter, under photometric conditions, were obtained. The images were observed with median seeing values of 0 !'73 and 0.55 in $g^{\prime}$ and $i^{\prime}$ filters respectively. Figure 1 shows 
the color composite image of the SDSS-CG-6 group. All galaxies with measured radial velocities are marked (see below).

All observations were processed with the Gemini IRAF package version 1.8 inside $\mathrm{IRAF}^{2}$ in a standard way. Calibrations of the magnitudes to the standard system were derived using observations of standard stars from Landolt (1992) field RU149.

Object detection and photometry were performed on the $i^{\prime}$-band image with the SExtractor program (Bertin and Arnouts 1996). MAG_AUTO was adopted as the total magnitude. Colors were derived by measuring fluxes inside a fixed circular aperture of 24 pixels $\left(1^{\prime \prime} 75\right)$ in both filters, corresponding to a physical aperture of $6.2 \mathrm{~h}_{70}^{-1} \mathrm{kpc}$ at the rest frame of the group. All objects with SExtractor stellarity flag $\leq 0.85$ were selected as galaxies. We estimate that the catalog is complete down to $i^{\prime}=23.5 \mathrm{mag}$, since the number counts start to turn over at this value. The final catologue contains the total magnitudes, colors and structural parameters for 409 galaxies brighter than $i^{\prime}=23.5\left(M_{i^{\prime}} \sim-16.7 \mathrm{mag}\right.$ at the distance of the group, with no k-correction).

Galaxies for spectroscopic follow-up were selected based on their magnitudes and colors. Figure2 2 shows the color-magnitude diagram for galaxies with $i^{\prime} \leq 23$ mag. A pronounced red sequence is readly seen. Objects in the region below the red cluster sequence (continuous line) and brighter than $i^{\prime}=21 \mathrm{mag}\left(M_{i^{\prime}}<-19.2\right.$, (dashed line) were selected as potential candidates of the system.

Galaxy spectra were observed in December 2005, during dark time, with relatively good transparency and with a seeing that varied between $0 . \prime 9$ and $1^{\prime \prime} .0$. Three exposures of 1400 seconds were obtained through a mask with 1..0 slits, using the 400 lines $\mathrm{mm}^{-1}$ ruling density grating (R400) and centered at $6300 \AA$, for a resolution of $8 \AA$. Reduction and calibration of the data were done in the standard way, using the Gemini IRAF package version 1.8 .

We were able to measure redshifts for 35 observed galaxies using cross-correlation techniques or emissionline fitting Twenty two of the galaxies are located at the redshift of the group (within $\pm 2000 \mathrm{~km} \mathrm{~s}^{-1}$ and between $0.213<\mathrm{z}<0.226$ ). We used the ROSTAT program (Beers at al. 1990) to calculate the average velocity and the one-dimensional line-of-sight velocity dispersion of the group. We found that the group is located at $\langle z\rangle=0.21981 \pm 0.00054$, with a velocity dispersion of $\sigma=703 \pm 103 \mathrm{~km} \mathrm{~s}^{-1}$, and with 20 member galaxies. Figure 3 shows the velocity distribution of the 20 galaxies at the redshift of the group. We used the same software to do a statistical analysis of the velocities and we found no large gap in the velocity distribution, which follows close to a gaussian shape. The virial mass computed using the 20 galaxies with concordant redshifts, for a distance modulus of 40.18 is $\mathrm{M}_{v i r}=1.77_{-0.21}^{+0.45} \times$ $10^{14} \mathrm{~h}_{70}^{-1} \mathrm{M}_{\odot}$ and the virial radius is $\mathrm{R}_{\text {vir }}=0.82_{-0.01}^{+0.16}$ $\mathrm{h}_{70}^{-1} \mathrm{Mpc}$ (the errors are $68 \%$ confidence intervals).

We checked for any significant effects in the velocity dispersion and total mass of the system when we exclude

\footnotetext{
2 IRAF is distributed by NOAO, which is operated by the Association of Universities for Research in Astronomy Inc., under contract with the National Science Foundationand
}

the emission line galaxies from the sample. The hashed histogram in Figure 3 shows the distribution of the 15 non-emission line galaxies. For this smaller sample, we obtained a lower velocity dispersion of $\sigma=608 \pm 99 \mathrm{~km}$ $\mathrm{s}^{-1}$, a virial mass of $\mathrm{M}_{v i r}=0.95_{-0.11}^{+0.32} \times 10^{14} \mathrm{~h}_{70}^{-1} \mathrm{M}_{\odot}$ and a virial radius $\mathrm{R}_{\text {vir }}=0.64_{-0.02}^{+0.18} \mathrm{~h}_{70}^{-1} \mathrm{Mpc}$ (again the errors are $68 \%$ confidence intervals), values not significantly different from those considering the whole sample. The results go in the direction of what was previously seen also for other systems: inclusion of the emission-line galaxies enhance the velocity dispersion of the system.

\section{DISCUSSION}

Dynamical friction and subsequent merging are probably the processes responsible for the lack of $\mathrm{L}^{*}$ galaxies in FGs. Considering the merging scenario, it is possible that the overluminous central galaxy in a FG has been formed within a substructure, inside a larger structure. In that case, one could think of a scenario where a CG was formed within a rich group, which would then have merged, leaving behind the brightest elliptical galaxy of what today is seen as a FG. One weak argument against this scenario is that the nearby examples of CGs are not usually found within such massive structures, but instead are more often surrounded by very sparse structures. There are, however, examples such as CG6, surrounded by large numbers of lower-luminosity galaxies, inhabiting a deep potential well.

We would like to test the hypothesis that CGs, as observed in the nearby universe, could be the precursors of FGs. We may examine two aspects: (1) if the sum of the luminosities of the brightest CG galaxies is similar to the luminosity of a first-ranked FG galaxy and; (2) if the neighbourhoods of CGs are rich, i.e., if the system as a whole (group plus environment) has a velocity dispersion/mass similar to that of a FG.

We compute the total luminosity of the galaxies in the soon-to-merge CGs, HCG 31, HCG 79 and HCG 92, to check how these compare with the luminosities of firstranked galaxies in FGs. Adding up the luminosities of galaxies $\mathrm{HCG} 31 \mathrm{~A}$ to $\mathrm{C}, \mathrm{G}$ and $\mathrm{Q}$, which are the brightest in the group $\mathrm{HCG} 31$, a magnitude of $\mathrm{M}_{R}=-22.5$ is obtained (for a distance modulus, DM = 33.8). Summing up the luminosities of galaxies HCG 79 A-D, an equal total magnitude of $\mathrm{M}_{R}=-22.5$ is obtained (for $\mathrm{DM}=34.0)$. These are upper limits on the luminosities of these objects given that several members are starburst galaxies. After fading, the merged central object in HCG 31 and HCG 79 will have somewhat lower magnitudes than that of a typical first-ranked galaxy in a FG. Fossil groups first-ranked galaxies have luminosities well above $\mathrm{L}^{*}$. For the five FGs studied by Jones et al. (2003), the first-ranked galaxies had a median luminosity of $M_{R}=-23.2$ and for the 34 FGs found in the SDSS DR5 by dos Santos et al. (2007) the median luminosity was $M_{R}=-23.5$.

Although for HCG 92, the final object (adding up luminosities of galaxies A-E) would have an absolute magnitude of $M_{R}=-24.2$ (for $\mathrm{DM}=34.8$ ), which after allowing for some fading, could be similar to that of an FG firstranked galaxy, HCG 92 would possibly still not resemble an FG when merged, because its neighbourhood is very sparse, i.e., it is not embedded in any larger structure, as it is often the case for the central galaxy in FGs. This 
is in agreement with its relatively low bolometric X-ray luminosity of $2.96 \times 10^{42} \mathrm{~h}_{70}^{-2}$ ergs s$^{-1}$ (Xue \& Wu 2000).

The environments of nearby CGs have been surveyed by Ribeiro et al. (1998); Zabludoff \& Mulchaev (1998); Carrasco et al. (2006) among others. Spectroscopy of dozens of members in the neighbourhood of quite a number of groups was obtained, confirming in all cases that CGs have low velocity dispersions typical of the group regime (typically $200-300 \mathrm{~km} \mathrm{~s}^{-1}$ ). In fact, even for HCG 62, thought to be one of the most massive CGs in Hickson's catalogue, the velocity dispersion obtained from 45 members of the system showed that it is a bonafide group $\left(376 \mathrm{~km} \mathrm{~s}^{-1}\right)$. HCG 62 was suggested by Ponman \& Bertram (1993) as an example of a system that could turn into a FG in a few Gygayears, but its velocity dispersion is still much lower than the value of $\sim 600 \mathrm{~km} \mathrm{~s}^{-1}$, typical for rich FGs. Two other massive nearby CGs in Hickson's catalogue are HCG 94 and HCG 65. The first is known to have a very high bolometric X-ray luminosity of $2.35 \times 10^{44} \mathrm{~h}_{70}^{-2}$ (Xue \& Wu 2000) which may, however, be contaminated by the emission of a nearby cluster. The velocity dispersion obtained from 11 members in this system gives a value of $479 \mathrm{~km} \mathrm{~s}^{-1}$. HCG 65 is the center of the cluster Abell 3559. It is in the heart of the Shapley supercluster and its location makes it hard to disentangle its dynamics and determine its mass. The three most massive Hickson CGs known, HCG 62, HCG 65 and HCG 94, are strongly early-type dominated, as expected from the ve- locity dispersion-morphology relation observed for CGs.

The conclusion is then that a typical CG, as observed at $\mathrm{z}=0$, is unlike to turn into a FG. It is more likely to merge into an isolated elliptical galaxy. For the compact group CG 6 , at $z=0.22$ and $\sigma=703 \mathrm{~km} \mathrm{~s}^{-1}$, if we merge the four central galaxies (A, B, C and D), we end up with a galaxy with total magnitude $i^{\prime}=16.31$ and $g^{\prime}=17.80 \mathrm{mag}\left(\mathrm{M}_{i^{\prime}}=-23.87\right.$ and $\mathrm{M}_{g^{\prime}}=-22.38$, with no k-correction). Using the color relation for a galaxy at $\mathrm{z}=0.2$ from Fukugita et al. (1995), the magnitude in $\mathrm{r}^{\prime}$ will be 16.83 or $\mathrm{M}_{r}=-23.35$. This magnitude is similar to those of typical central galaxies in FGs and the velocity dispersion of the system is typical for the studied FGs. However, no gap of at least two magnitudes between the first-ranked relic and the remaining objects of the system would be observed because there is at least one other bright galaxy in the system within half the virial radius of the group. We point out that one other example of a possible massive system, at $\mathrm{z}=0.39$, which may turn into a FG, has recently been discovered by Rines et al. (2007). Spectroscopic studies of CGs at medium redshifts may find many more of such objects.

We acknowledge support from the Brazilian agencies FAPESP (projeto temático 01/07342-7), CNPq and CAPES. We thank Laerte Sodré Jr. and Eduardo Cypriano for useful discussions in the earlier phase of this project.

\section{REFERENCES}

Amram, P., Mendes de Oliveira, C., Plana, H., Balkowski, C. Hernandez, O., Carignan, C. et al. 2004, ApJ, 612, L5

Amram, P., Mendes de Oliveira, C., Plana, H., Balkowski, C. \& Hernandez, O. 2007, A\&A, 471, 753

Barnes, J. E. 1992, ApJ, 393, 484

Beers, T. C., Flynn, K. \& Gebhardt, K. 1990, AJ, 100, 32

Bertin, E. \& Arnouts, S. 1996, A\&AS, 117, 393

Carrasco, E. R., Mendes de Oliveira, C. \& Infante, L. 2006, AJ, 132,1796

Cypriano, E. S., Mendes de Oliveira, C. L., Sodré Jr., L. 2006, AJ, 132,514

Da Rocha, C., de Oliveira, C. Mendes 2005, MNRAS, 364, 1069

dos Santos, W., Mendes de Oliveira, C. \& Sodré Jr., L. 2007, AJ, 134,1551

Fukugita, M., Shimasaku, K. \& Ichikawa, T. 1995, PASP,

Fukugita, M., Ichikawa, T., Gunn, J. E., Doi, M., Shimasaku, K. \& Schneider, D. P. 1996, AJ, 111, 1748

Hickson, P., Mendes de Oliveira, C., Huchra, J. P., Palumbo, G. G. 1992, ApJ, 399, 353

Hook, I., Jørgensen, I., Allington-Smith, J. R., Davies, R. L., Metcalfe, N., Murowinski, R. G., Crampton, D. 2004, PASP, 116, 425

Jones, L. R., Ponman, T. J., Horton, A., Babul, A., Ebeling, H., Burke, D. J. 2003, MNRAS, 343, 627

Khosroshahi, H.G., Maughan, B.J., Ponman, T.J. \& Jones, L.R., 2006, MNRAS, 369, 1211

Landolt, A.U. 1992, AJ, 104, 340
Lee, B. C., Allam, S. S., Tucker, D. L., Annis, J., Johnston, D. E., Scranton, R., et al. 2004, AJ, 127, 1811

Mendes de Oliveira, C. \& Hickson P. 1991, ApJ, 380, 30

Mendes de Oliveira, C., Plana, H., Amram, P., Balkowski, C., Bolte, M. 2001, AJ, 121, 2524

Mendes de Oliveira, C., Cypriano, E. S., Sodré., Jr., Balkowski, C, 2004, ApJ, 605, 17

Mendes de Oliveira, C. L., Cypriano, E. S., Sodré Jr., L. 2006a, AJ, 131, 158

Mendes de Oliveira, C. L., Temporin, S., Cypriano, E. S., et al., 2006b, AJ, 132, 570

Pildis, R. A., Bregman, J. N., Evrard, A. E. 1995, ApJ, 443, 514

Ponman, T. J. \& Bertram, D. 1993, Nature, 363, 51

Ribeiro, A. L. B. et al. 1998, ApJ, 497, 72

Rines, K., Finn, R. and Vikhlinin, A. 2007, ApJ, 665, 9

Verdes-Montenegro, L., Del Olmo, A., Yun, M. S., \& Perea, J. 2005, A\&A, 430, 443

Vikhlinin, A., McNamara, B. R., Hornstrup, A., Quintana, H., Forman, W. et al., 1999, ApJ, 520, 1

Williams, B. A., McMahon, P. M. \& van Gorkom, J. H. 1991, AJ, 101,1957

Xu, C. Kevin et al. 2005, ApJ, 619, 95

Xue, Y. J. \& Wu, X. P. 2000, ApJ, 538, 65

Zabludoff, A. I., Mulchaey, J. S. 1998, ApJ, 498, 5

Zepf, S. E. 1993, ApJ, 407, 448 


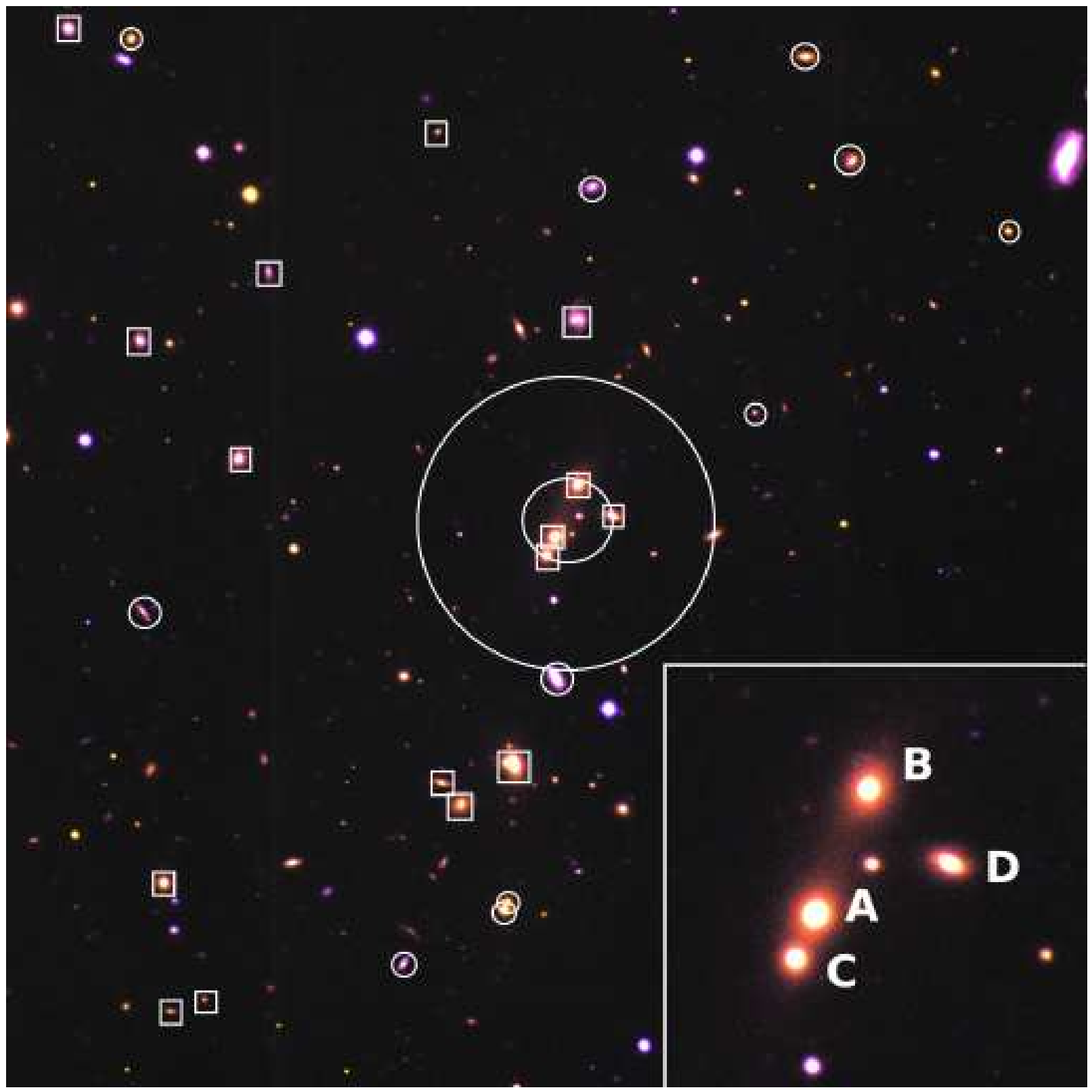

FIG. 1. - Color composite image of SDSS-CG-6. The field of view is 5!15 on a side $\left(1.12 \mathrm{~h}_{70}^{-1} \mathrm{Mpc}\right.$ at the distance of the group). The squares are galaxies members of the group. The circles are galaxies in the foreground and background of the group. The inner circle is the smallest circle that contains the centers of the group members with magnitudes within 3 mag from the brightest group member. The outer concentric circle corresponds to the angular diameter that contains no other (external) galaxies within 3 mag from the brightest group member, as defined by Lee et al. (2004). The zoom (inner $0.2 \mathrm{~h}_{70}^{-1} \mathrm{Mpc}$ ) shows the four central galaxies in the group. 


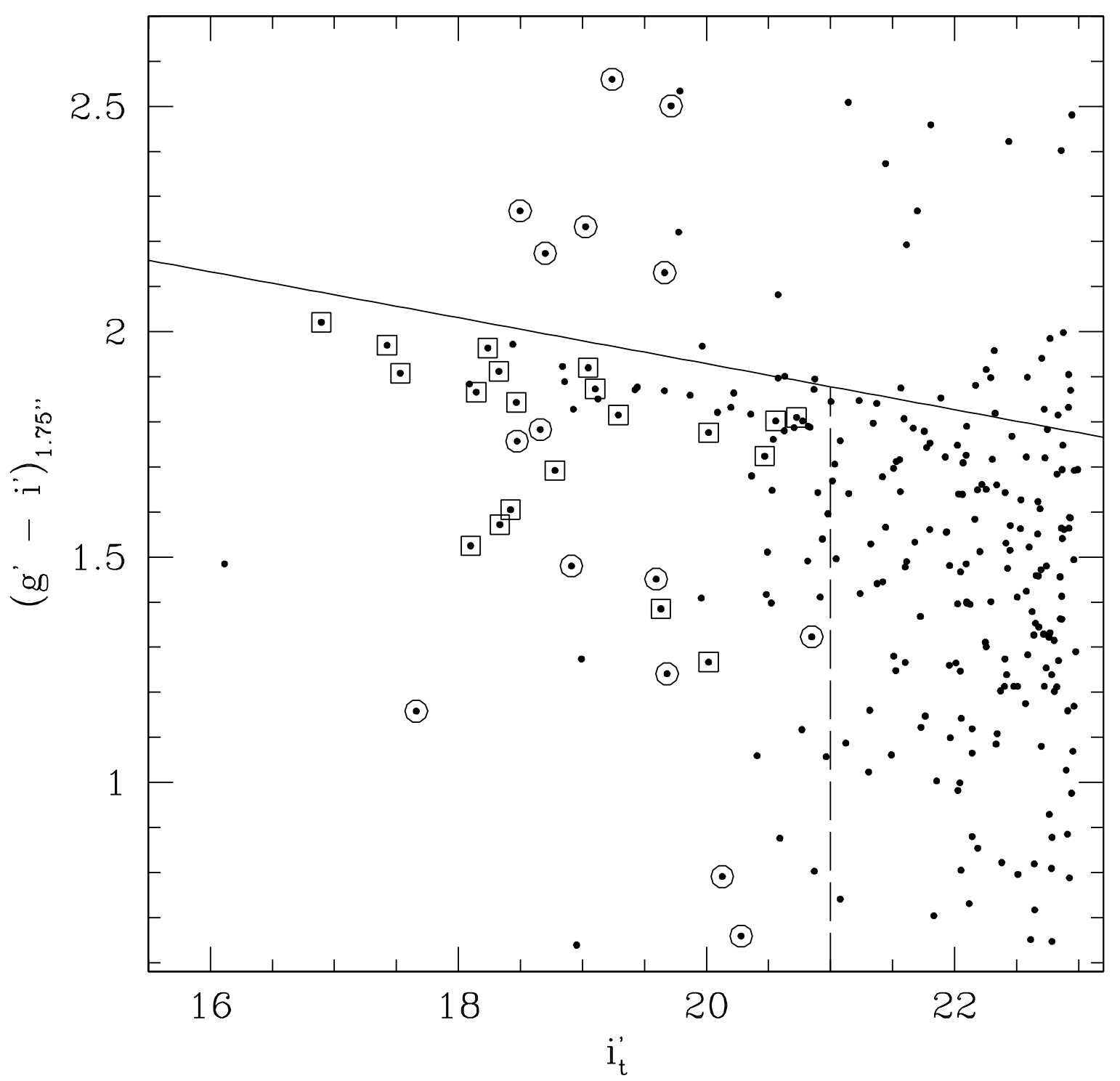

FIG. 2.- Color-magnitude diagram of all galaxies brighter than 23 mag in the CG 6 field (dots). Colors and magnitude are corrected for galactic extinction. The symbols are the same as in Figure 1 The solid and dashed lines indicate the upper limit for the red sequence and the limiting magnitude we adopted for the spectroscopic target selection. 


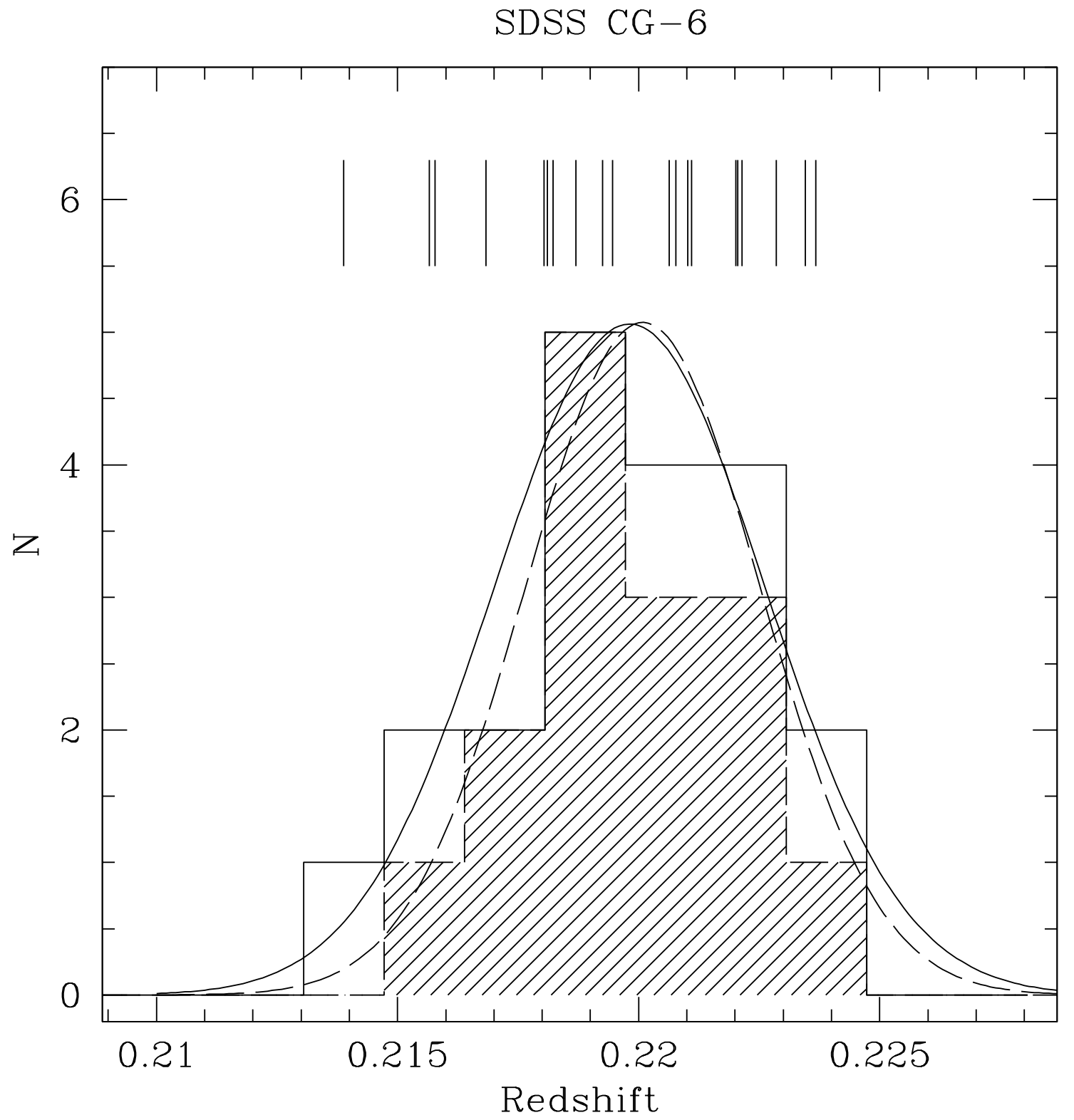

FIG. 3.- Histogram of the redshift distribution of 20 galaxies in area of SDSS-CG-6. The average redshift and the velcity dispersion for the whole sample (20 galaxies) is $\langle z\rangle=0.21981$ and $\sigma=703 \mathrm{~km} \mathrm{~s}^{-1}$ (continuous line). The hashed histogram show the velocity distribution for the 15 non-emission line galaxies in the sample. For this sub-sample, the average redshift is $\langle z\rangle=0.21837$ and the velocity dispersion is $\sigma=608 \mathrm{~km} \mathrm{~s}^{-1}$ (dashed line). 